\title{
Cellulose Sulfuric Acid: An Efficient and Recyclable Solid Acid Catalyst for the Protection of Hydroxyl Groups Using HMDS under Mild Conditions
}

\author{
HAMID REZA SHATERIAN*, FATEMEH RIGI and MADIHE ARMAN \\ Department of Chemistry, Faculty of Sciences, University of Sistan and Baluchestan, \\ PO Box 98135-674, Zahedan, Iran \\ hrshaterian@chem.usb.ac.ir
}

Received 7 May 2012 / Accepted 17 May 2012

\begin{abstract}
Primary, secondary and tertiary alcohols as well as phenols and naphthols were effectively converted into their corresponding trimethylsilyl ethers with hexamethyldisilazane in the presence of cellulose sulfuric acid as a biopolymer-based solid acid catalyst under very mild conditions at room temperature with short reaction times in good to excellent yields.
\end{abstract}

Keywords: Trimethylsilylation, Hexamethyldisilazane, Cellulose sulfuric acid, Solvent-free

\section{Introduction}

Cellulose is one of the most abundant natural materials in the world and it has been widely studied in organic transformations ${ }^{1}$. Cellulose constitutes the most abundant renewable polymer resource available today. As a chemical raw material, it is generally well-known that it has been used in the form of fibers or derivatives for nearly 150 years for a wide spectrum of products and materials in daily life ${ }^{2}$. Cellulose is potential as a biodegradable material, can be used for several applications and also as support for bonding several functional groups which act as catalysts to yield clean efficient and fast chemical reactions ${ }^{3}$. Cellulose sulfuric acid (CellSA) can be easily prepared by the reaction of inexpensive cellulose with chlorosulfonic acid (Figure 1$)^{4}$.

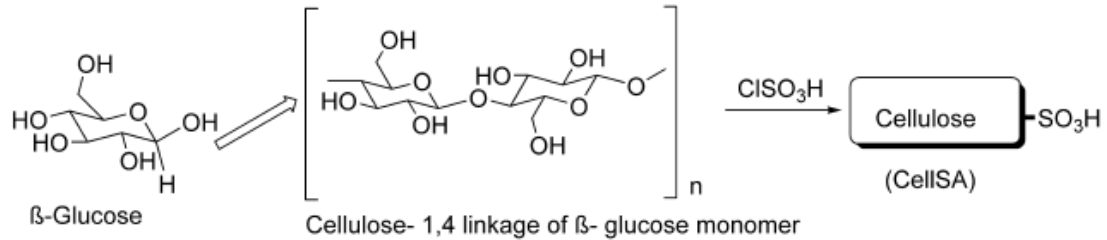

Figure 1. Preparation of cellulose sulfuric acid (CellSA)

The number of acidic $\left(\mathrm{H}^{+}\right)$sites in the cellulose sulfuric acid is $0.50 \mathrm{meq} / \mathrm{g}$ in the basis of acid-base titration ${ }^{4}$. Cellulose sulfuric acid (CellSA) has excellent catalytic properties, which are attributed to the high thermal stability and strong acid sites of sulfonic acid functional groups. CellSA as a non-hygroscopic solid acid catalyst acts as an efficient and environmentally 
benign catalyst for the synthesis of several organic compounds such as 3,3'-indolyloxindole derivatives $^{5}$, 2,4,5-triarylimidazoles ${ }^{6}, \beta$-acetamido carbonyl derivatives ${ }^{7}$, Knoevenagel condensation $^{8}$, oxazolines, imidazolines and thiazolines ${ }^{9}$.

Trimethylsilyl protection of alcohols has already been recognized as an important part of organic, inorganic and analytical chemistry ${ }^{10}$. This synthetic methodology was used especially as protecting group approach in many multi-steps syntheses with reasonable complexity for masking hydroxyl functional groups ${ }^{11}$. This transformation enhances solubility in non-polar solvents, low viscosity, increases thermal stability, resistant to oxidation and in addition trimethylsilylation of hydroxyl compounds is used extensively to increase volatility of the compounds for gas chromatography and mass spectrometry as well ${ }^{12}$.

The preparation of silyl ethers could carry out by treatment of alcohols with silyl chlorides in the presence of stoichiometric amount of an organic base $\mathrm{e}^{13}$ or silyl triflates with $\mathrm{R}_{3} \mathrm{Si}-\mathrm{H}$ activated by dirhodium(II) perfluorooctanoate ${ }^{14}$. However, these methods suffered from drawbacks such as lack of reactivity or the difficulty in removal of amine salts ${ }^{13,14}$. 1,1,1,3,3,3- Hexamethyldisilazane (HMDS) as an inexpensive and easy available compound has been used for the preparation of trimethylsilyl ethers from hydroxyl compounds ${ }^{15}$. The only by product of this reaction is $\mathrm{NH}_{3}$ gas, which is a notable advantage for the method ${ }^{15}$. Even though the handling of this reagent is easy, but the low silylation power of HMDS is the main drawback to its application ${ }^{16}$ therefore, there are a variety of catalysts for activating of this reagent, such as zirconium sulfophenyl phosphonate ${ }^{16}$, Envirocat EPZGO ${ }^{17}$,

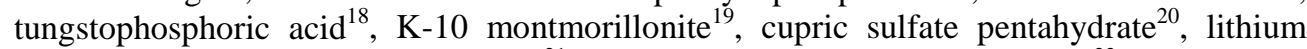
perchlorate supported on silica gel $^{21}$, sulfonic acid-functionalized silica ${ }^{22}$, magnesium triflate $^{23}$, alumina-supported heteropolyoxometalates ${ }^{24}$, iron(III) trifluoroac ${ }^{28}$ and $\mathrm{TiCl}_{2}(\mathrm{OTf})-\mathrm{SiO}_{2}{ }^{29}$. Recently, an uncatalyzed method for the silylation of alcohols and phenols with HMDS in $\mathrm{CH}_{3} \mathrm{NO}_{2}$ was reported ${ }^{30}$. Even though these catalytic systems enhance the ability of HMDS for the silylation, still some of the catalysts require long reaction time, high temperature and excess amount of reagent. But the lack of facile and general synthetic methodology under essentially mild reaction condition has prompted us to develop an efficient, convenient and practical procedure for the silylation of alcohols under solvent-free conditions at room temperature. In the present research for functional group transformation, we wish to describe a new protocol for the mild and rapid trimethylsilylation of a wide variety of hydroxyl groups using HMDS in the presence of cellulose sulphuric acid (CellSA) as a recyclable and biodegradable solid acid catalyst at room temperature.

$$
\begin{gathered}
\mathrm{ROH}+\mathrm{HMDS} \frac{\text { CellSA (Cat) }}{\text { r.t, }} \text { ROSiMe }_{3}+\mathrm{NH}_{3} \\
\text { Solvent-free }
\end{gathered}
$$

\section{Experimental}

\section{Scheme 1}

All reagents were purchased from Merck and Aldrich and used without further purification. CellSA was prepared according to the reported procedure ${ }^{4}$. All yields refer to isolated products after purification. Products were characterized by comparison with authentic samples and by spectroscopy data (FT-IR, ${ }^{1} \mathrm{H}$ NMR and ${ }^{13} \mathrm{C}$ NMR spectra). The NMR spectra were recorded on a Bruker Avance DPX $500 \mathrm{MHz}$ instrument. The spectra were measured in $\mathrm{CDCl}_{3}$ relative to TMS $(0.00 \mathrm{ppm})$. FT-IR spectra were recorded on a JASCO FT-IR 460 plus spectrophotometer. TLC was performed on Silica-gel polygram SIL G/UV 254 plates. 


\section{General procedure for trimethylsilylation of alcohols using HMDS}

To a stirred solution of alcohols (10 mmol) and HMDS (8 mmol) was added CellSA ( $0.7 \mathrm{~g})$ and the mixture was stirred at room temperature for the time specified in Table 1 . The reaction was monitored by TLC ( $n$-Hexane-EtOAc, 9:1). After completion of the reaction, the mixture was diluted with $n$-hexane. The resulting mixture was passed through a short pad of silica gel. Then, the pad column was washed with $n$-hexane $(2 \times 10 \mathrm{~mL})$. Evaporation of the solvent under reduced pressure gave pure product(s). The desired pure product(s) was characterized by comparison of their physical data with those of known compounds ${ }^{25,26,31}$. Selected spectral data for trimethylsilyation of benzyl alcohol: Trimethyl(benzyloxy)silane (Table 2, Entry 1): colorless liquid, ${ }^{1} \mathrm{H} \mathrm{NMR}\left(\mathrm{CDCl}_{3}, 500 \mathrm{MHz}\right): \delta=7.36-7.35(5 \mathrm{H}, \mathrm{m}), 4.72$ (2H, s), 0.18 (9H, s) ppm; ${ }^{13} \mathrm{C} \mathrm{NMR}\left(\mathrm{CDCl}_{3}, 125 \mathrm{MHz}\right): \delta=138.32,128.36,127.45,127.35$, 67.85, 0.29.; FT-IR $\left(\mathrm{CCl}_{4}\right)$ : 2957, 1496, 1454, 1377, 1250, 1207, 1096, 1027, 842, 727, $695 \mathrm{~cm}^{-1}$.

\section{Results and Discussion}

To optimize the amount of the alcohol, HMDS and CellSA as catalyst in the mentioned reaction, we have carried out the reaction of benzyl alcohols $(1 \mathrm{mmol})$ with HMDS $(0.8 \mathrm{mmol})$ in the presence of different amount of CellSA as catalyst under solvent-free conditions at room temperature as a model. It was found that $(0.07 \mathrm{~g}, 3.5 \mathrm{~mol} \%)^{4}$ of the CellSA as catalyst showed maximum yield (95\%) in minimum reaction time (3 min).

Using these optimized reaction conditions, the scope and efficiency of the reaction were explored for the synthesis of a wide range of structurally diverse and functionalized alcohols, phenols and naphthols at room temperature (Table 1). Generally, in the all cases of benzylic alcohols, primary, secondary and tertiary alcohols, phenols and naphthols were converted to corresponding trimethylsilyl ethers within less than 13 min by evolution of $\mathrm{NH}_{3}$ gas from the reaction mixture. There was not much difference in reactivity with variation of aliphatic and aromatic compounds containing hydroxyl functional groups. Only tertiary alcohols (Table 1, Entries 9, 10) took longer time to complete the reaction which might be due to steric hindrance. Phenols containing amino functional group were regioselectively $O$-silylated (Table 1, Entry 12), whereas amino group was intact. This is because oxophilicity of silicon atom towards alcoholic $\mathrm{OH}$ may be strong enough to overcome the reactivities of HMDS with $\mathrm{N}-\mathrm{H}$ of amines.

Table 1. Trimethylsilylation of benzylic alcohols, primary, secondary and tertiary alcohols, phenols and naphthols with HMDS in the presence of cellulose sulfuric acid as catalyst (0.07 g) under ambient conditions

\begin{tabular}{|c|c|c|c|c|c|}
\hline Entry & Substrate & Product & Time, min & $\begin{array}{c}\text { Yield, } \\
\%^{\mathrm{a}}\end{array}$ & $\begin{array}{c}\text { Literature } \\
\text { references for } \\
\text { known product }\end{array}$ \\
\hline 1 & & & 3 & 95 & 25,26,31 \\
\hline 2 & & & 8 & 95 & 31 \\
\hline 3 & & & 5 & 94 & 26 \\
\hline
\end{tabular}




(26,

${ }^{a}$ The molar ratio of substrate/HMDS was chosen 1/0.8. Yields refer to the pure isolated products. The structure all known products were confirmed by comparison of their spectral data (FT-IR, ${ }^{1} \mathrm{H} N M R$, ${ }^{13} \mathrm{C} \mathrm{NMR}$ ) with those of known samples in the litrature ${ }^{25,26,31}$

In all reactions, in the basis of observation fast evolution of ammonia gas and according to the literature ${ }^{26}$, we have proposed a mechanism in which the generation of $\mathrm{NH}_{3}$ and the role of CellSA in a catalytic cycle are clarified (Scheme 2). 


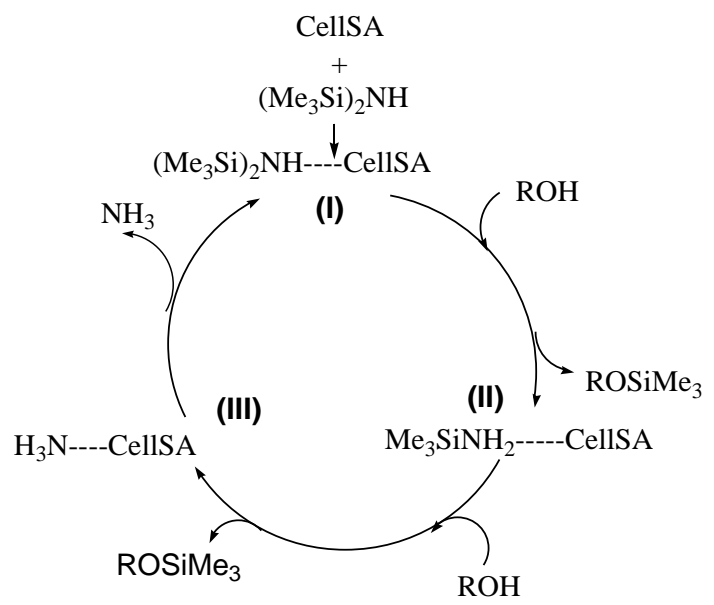

Scheme 2

In this mechanism, we have suggested that an acid-base interaction between catalyst and nitrogen in HMDS polarizes N-Si bond of HMDS to produce a reactive silylating agent (I). A rapid reaction with alcohol then ensues, leading to the ammonium silylating species (II) with concomitant release of the corresponding silyl ether. Irreversible cleavage of (II) with alcohol, leading to the fast evolution of $\mathrm{NH}_{3}$ and also formation of the unstable protonated silyl ether (III). Release of $\mathrm{H}^{+}$as catalyst from intermediate (III), re-enters catalytic cycle (Scheme 2).

The generality and the excellence of CellSA in terms of catalyst loading, short reaction time and considerably high yield can be easily understood from the comparison of the data with literature results as shown in Table 2.

Table 2. Comparison result of CellSA with trichloroisocyanuric $\operatorname{acid}^{32}, \operatorname{Mg}(\mathrm{OTf})_{2}{ }^{23}$, Lanthanum trichloride ${ }^{31}$ and alumina-supported heteropolyoxometalates ${ }^{24}$ in the silylation of benzyl alcohol with HMDS

\begin{tabular}{ccccc}
\hline Entry & Catalyst & Conditions & Time, min & Yield, \% \\
\hline 1 & $\begin{array}{c}\text { Trichloroisocyanuric acid } \\
0.06-0.1 \text { mmol }\end{array}$ & $\mathrm{CH}_{2} \mathrm{Cl}_{2}$, r.t & 240 & 90 \\
2 & $\begin{array}{c}\text { Lanthanum trichloride 0.2 mmol } \\
3\end{array}$ & $\begin{array}{c}\text { Alumina-supported } \\
\mathrm{CH}_{2} \mathrm{Cl}_{2} \text {, r.t }\end{array}$ & 180 & 91 \\
4 & $\begin{array}{c}\text { Toluene, r.t } \\
\text { heteropolyoxometalates 100 mg }\end{array}$ & 60 & 95 \\
5 & $\begin{array}{c}\text { Mg(OTf })_{2} \text { 0.1 eq } \\
\text { CellSA (0.07 g, 3.5 mol \%) }\end{array}$ & Neat, r.t & 38 & 95 \\
\hline
\end{tabular}

The recyclability of the catalyst in the reaction of benzyl alcohol, HMDS in the presence of cellulose sulfuric acid was checked (Table 1, Entry 1). The separated catalyst can be reused after washing with $n$-hexane and drying at $100{ }^{\circ} \mathrm{C}$. The catalyst was recovered in excellent yields and the catalyst was used in the mentioned reaction five times. It showed the same activity as fresh catalyst without any loss of its catalytic activity (Figure 2). 


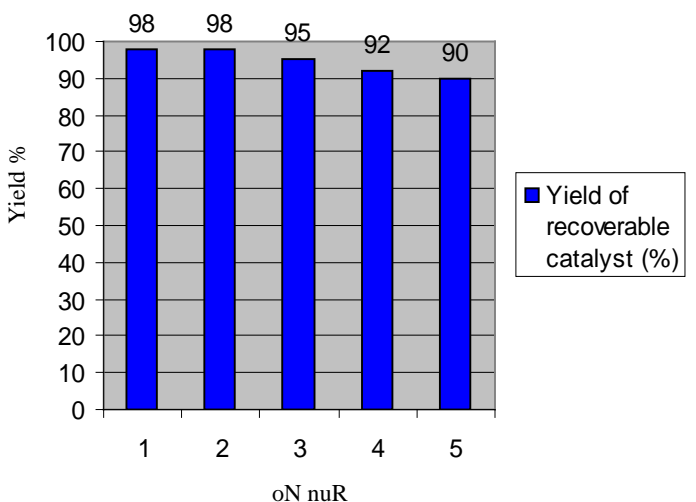

\section{Conclusion}

Figure 2

In summary, we have demonstrated that cellulose sulfuric acid is a new, efficient and reusable catalyst for trimethylsilylation of a variety of hydroxyl groups using HMDS under solvent-free, mild and ambient conditions. The reactions were carried out at room temperature with short reaction time and produce the corresponding trimethylsilyl ethers in good to excellent yields. The catalyst can be recovered from reaction mixtures by a simple filtration procedure.

\section{Acknowledgement}

We are thankful to the University of Sistan and Baluchestan Research Council for the partial support of this work.

\section{References}

1. Brown R M and Saxena I M, Cellulose: Molecular and Structural Biology: Selected Articles On The Synthesis, Stracture and Application Of Cellulose; Springer: Netherlands, 2007.

2. Habibi Y, Lucia L A and Rojas O J, Chem Rev., 2010, 110, 3479-3500.

3. Molvinger K, Quignard F, Brunel D and Boissiere M J M, Chem Matter., 2004, 16, 3367-3372.

4. $\quad$ Shaabani A and Maleki A, Appl Catal A., 2007, 331, 149-151.

5. $\quad$ Alinezhad H, Haghigh A and Salehian F, Chin Chem Lett., 2010, 21, 183-186.

6. Shelke K F, Sapkal S B, Kakade G K, Bapurao B and Shingare M S, Green Chem Lett Rev., 2010, 3, 27-32.

7. Oskooie H A, Heravi M M, Tahershamsi L, Sadjadi S and Tajbakhsh M, Synth Commun., 2010, 3, 1772-1777.

8. Shelke K F, Sapkal S B, Niralwad K S, Shingate B B and Shingare M S, Cent Eur J Chem., 2010, 8,12-18.

9. Shaabani A, Seyyedhamzeh M, Maleki A and Rezazadeh F, Appl Catal A, 2009, 358, 149-151.

10. Hanson J R, Organic Synthetic Methods; The Royal Society of Chemistry: Cambridge England, 2002.

11. (a) Wuts P G M and Greene T W, Greene's Protective Groups in Organic Synthesis, $4^{\text {th }}$ Ed; John Wiley and Sons: New Jersey, 2007 (b) Brook M A, Silicon in Organic, Organometallic and Polymer Chemistry; Wiley: New York , 2000. 
12. (a) Kocienski P J, Protecting Groups, $3^{\text {th }}$ Ed., George Tieme Verlag: Germany, 2005.

(b) Van Look G, Simchen G and Heberle J, Silylation Agents; Fluka Chemie, Buchs: Switzerland, 1995.

13. (a) Morita T, Okamoto Y and Sakurai H, Tetrahedron Lett., 1980, 21, 835-838. (b) Vesoglu T and Mitscher L A, Tetrahedron Lett., 1981, 22, 1299-1302.

14. Bifiss A, Castello E, Zecca M and Basato M, Tetrahedron., 2001, 57, 10391-10394.

15. Rajagopal G, Lee H and Kim S S, Tetrahedron., 2009, 65, 4735-4741.

16. Curini M, Epifano F, Marcotullio M C, Rosati O and Costantino U, Synth Commun., 1999, 29, 541-546.

17. Bandgar B P and Wadgaonkar P P, Synth Commun., 1997, 27, 2069-2074.

18. Firouzabadi H, Iranpoor N, Amani K and Nowrouzi F, J Chem Soc Perkin Trans 1., 2002, 1, 2601-2604.

19. Zhang Z H, Li T S, Yang F and Fu C G, Synth Commun., 1998, 28, 3105-3114.

20. Akhlaghinia B and Tavakoli S, Synthesis, 2005, 11, 1775-1778.

21. Azizi N, Yousefi R and Saidi M R, J Organomet Chem., 2006, 691, 817-820.

22. Zareyee D and Karimi B, Tetrahedron Lett., 2007, 48, 1277-1280.

23. Firouzabadi H, Iranpoor N, Sobhani S and Gassamipour S, J Organomet Chem., 2004, 689, 3197-3202.

24. Villabrille P, Romanelli G, Quaranta N and Vázquez P, Appl Catal B., 2010, 96, 379-386.

25. Firouzabadi H, Iranpoor N, Jafari A A and Jafari M R, J Organomet Chem., 2008, 693, 2711-2714.

26. Shaterian H R, Shahrekipoor F and Ghashang M, J Mol Catal A: Chem., 2007, 272, 142-151.

27. Mojtahedi M M, Abaee M S and Eghtedari M, Appl Organometal Chem., 2008, 22, 529-532.

28. Khazaei A, Zolfigol M A, Tanbakouchian Z, Shiri M, Niknam K and Saien J, Catal Commun., 2007, 8, 917-920.

29. Firouzabadi H, Iranpoor N and Farahi S, J Organomet Chem., 2009, 694, 3923-3928.

30. Kadam S T and Kim S S, Green Chem., 2010, 12, 94-98.

31. Narsaiah A V, J Organomet Chem., 2007, 692, 3614-3618.

32. Khazaei A, Zolfigol M A., Rostami A and Ghorbani Choghamarani A, Catal Commun., 2007, 8, 543-547. 